\title{
Avaliação do Estado de Fluxo e do Aprendizado em Atividades Desplugadas no Ensino do Pensamento Computacional com Estudantes do Ensino Médio
}

\author{
Leonardo S. e Silva ${ }^{1}$, Elmano R. Cavalcanti ${ }^{1}$ \\ ${ }^{1}$ Coordenação de informática - Instituto Federal de Pernambuco (IFPE) \\ Garanhuns - PE - Brazil \\ \{leonardo.silva, elmano.cavalcanti\}@garanhuns.ifpe.edu.br
}

\begin{abstract}
Statistical evidence is needed to prove the effectiveness of teaching computational thinking without the use of computers (CS Unplugged). This study aims to evaluate two unplugged activities from the official website, regarding the effectiveness in promoting the flow state (Flow Theory) and learning of computational thinking. A quasi-experiment was conducted with 35 high school students. The results suggest that the evaluated activities have potential to stimulate the flow state in students, even if they have an age group above the official recommendation. On the other hand, no statistical correlation was observed between this state and the learning of computational thinking.
\end{abstract}

Resumo. Evidências estatísticas são necessárias para comprovar a efetividade do ensino do pensamento computacional sem o uso do computador (CS Unplugged). Este estudo tem por objetivo avaliar duas atividades "desplugadas" do site oficial, quanto à efetividade na promoção do estado de fluxo (Flow Theory) e promoção do conhecimento sobre o pensamento computacional. Um quasi-experimento foi realizado com 35 estudantes do ensino médio. Os resultados sugerem que as atividades apresentam potencial para estimular o estado de fluxo nos estudantes. Por outro lado, não foi observada correlação estatística entre esse estado e o aprendizado do pensamento computacional.

\section{Introdução}

A promoção do aprendizado sobre o pensamento computacional sem o uso de computadores (prática denominada CS Unplugged) é uma importante estratégia para inclusão deste tema na educação de crianças e jovens [Bell et al. 2011]. A proposta envolve atividades lúdicas que trabalham conceitos da computação, ao mesmo tempo que constrói um importante espaço social de interação entre os estudantes. Não utilizar computadores é um meio para desconstruir certos preconceitos associados à área, como também torna o ensino mais democrático, uma vez que alguns estudantes possuem dificuldades no manuseio destes equipamentos e isto poderia prejudicá-los no processo de aprendizagem.

Para que professores sintam-se confiantes em adotar esta estratégia pedagógica, é necessário que estudos empíricos validem a eficácia das atividades de computação desplugadas. Um quasi-experimento foi realizado com 35 estudantes de 13 a 16 anos. Os resultados indicam que as atividades proporcionaram um elevado estado de fluxo, e indicam que os estudantes engajaram-se nas atividades. Por outro lado, não foi observada correlação estatisticamente significante entre o estado do fluxo dos estudantes e seus desempenhos na avaliação de conhecimento. 
VII Congresso Brasileiro de Informática na Educação (CBIE 2018)

Anais do XXIX Simpósio Brasileiro de Informática na Educação (SBIE 2018)

\section{Trabalhos relacionados}

Pesquisadores buscaram avaliar as atividades desplugadas sob diferentes perspectivas. Uma das linhas de investigação preocupou-se em avaliar o conhecimento dos estudantes, a partir de intervenções com essas atividades ([Rodriguez et al. 2017]; [Brackmann et al. 2017a]; [Costa 2017], ao qual também se inclui esta pesquisa. Citamos como principal diferença do nosso estudo para os trabalhos mencionados, a avaliação de duas atividades desplugadas que não foram adotadas por eles.

Por fim, alguns estudos relataram que as atividades desplugadas motivam os estudantes, porém não utilizaram instrumentos específicos para medi-la ([Brackmann et al. 2017b]; [Curzon et al. 2009]). Um estudo similar ao nosso, buscou medir a motivação intrínseca de estudantes que utilizaram atividades desplugadas [Jiang and Wong 2018]. Os resultados indicam que os estudantes sentiram-se motivados com a intervenção. Diferimos do referido estudo por avaliar o estado de fluxo, que apesar de apresentar relação com a motivação [Mills and Fullagar 2008], representa um conceito mais amplo.

\section{Metodologia}

\subsection{Caracterização do contexto pedagógico e amostra}

Um quasi-experimento foi realizado com estudantes do curso técnico em informática integrado ao ensino médio do Instituto Federal de Pernambuco - campus Garanhuns. Trinta e cinco estudantes participaram do experimento. Os estudantes apresentavam faixa-etária entre 13 a 16 anos, com média de 14,8 e desvio padrão de 0,97. Sendo 22,9\% do gênero feminino e $77,1 \%$ do gênero masculino. $98 \%$ nunca tiveram contato anterior com programação e aproximadamente $30 \%$ não possuem computador em casa.

As atividades desplugadas ocorreram em sala de aula, como parte curricular da disciplina de introdução à programação. Trata-se de uma matéria que é lecionada semanalmente por $1 \mathrm{~h} 30$ minutos, durante o período de um ano letivo, totalizando 60 horas de aulas. Nessa disciplina os alunos trabalham os conceitos de pensamento algorítmico, repetições, condições, variáveis, funções e arrays, utilizando a linguagem de programação Java.

As atividades de CS Unplugged ocorreram na terceira e quarta semana de aula, após a introdução à temática de programação de computadores que ocorreu na primeira aula. As atividades seguiram o modelo proposto no site oficial do projeto http://csunplugged.org/.

Para avaliar o estado de fluxo, utilizou-se o Flow Short Questionnaire, descrito em [Rheinberg et al. 2003]. Em razão do questionário ser no idioma inglês, utilizou-se a tradução utilizada no trabalho de doutorado [Moura Júnior et al. 2015]. Este questionário é formado por 10 questões que buscam compreender a percepção do sujeito sob uma determinada atividade. Utiliza-se uma escala de Likert com 7 escalas, e o resultado final do questionário varia de 0 a 7 , no qual 0 significa ausência de engajamento do estudante e 7 que o estudante estava completamente imerso na atividade. A vantagem deste questionário sobre outros que também buscam medir o estado de fluxo é o seu tamanho reduzido que facilita a aplicação, em especial com adolescentes. Cada uma das questões do questionário foi lida pelo professor e discutida com os estudantes sobre o seu objetivo, como também eventuais esclarecimentos. 
VII Congresso Brasileiro de Informática na Educação (CBIE 2018)

Anais do XXIX Simpósio Brasileiro de Informática na Educação (SBIE 2018)

\subsection{Descrição da intervenção}

\subsubsection{Ensino de números binários}

A primeira atividade envolveu o conhecimento sobre números binários e seguiu a atividade How Binary Digits Works?. Os estudantes foram apresentados brevemente ao tema, sobre a importância da numeração binária para a computação e em seguida iniciou-se a atividade seguindo as instruções do site.

Para avaliar os conhecimentos dos estudantes em números binários, uma avaliação com oito questões foi realizada após a intervenção. Os alunos deveriam converter números em binário para inteiro e de inteiro para binário. A atividade foi realizada individualmente e os estudantes tiveram 20 minutos para responder as questões, utilizando papel e caneta.

\subsection{Ensino do pensamento algorítmico}

A segunda atividade proposta, baseou-se na atividade Sending a Rocket to Mars. A mudança realizada em relação à atividade original é que ela foi aplicada individualmente, ao invés de ser uma atividade em grupo. Nessa atividade, o estudante é apresentado a um tabuleiro contendo 8 linhas por 8 colunas, devendo controlar um foguete que sai da terra em direção a Marte, sendo necessário desviar de obstáculos existentes no trajeto e seguir a rota de acordo com a disposição destes e do planeta no tabuleiro. O controle é feito por meio de setas: cima, baixo, esquerda, direita, virar esquerda e virar direita.

Foram utilizados três tabuleiros diferentes, em que o primeiro é mais simples e o último apresenta mais obstáculos e uma distância maior entre o foguete e Marte. Os estudantes escreveram as setas com papel e caneta.

As respostas foram avaliadas observando a quantidade de setas utilizadas em relação a um modelo considerado ideal (havendo o uso de $30 \%$ mais setas que o modelo ideal, o aluno iria perder $50 \%$ da nota), e se ao término dos comandos o foguete alcançou o planeta, não alcançando, o aluno iria perder $50 \%$ da nota.

\subsection{Formulação de hipóteses e planejamento estatístico}

Duas questões de pesquisa foram elencadas: i) qual o estado de fluxo apresentado pelos estudantes do ensino técnico integrado ao ensino médio em informática, em atividades desplugadas? e ii) há correlação entre o fluxo apresentado pelos estudantes nas atividades e o desempenho em atividades de avaliação do conhecimento?

Duas hipóteses de investigação foram elencadas: $\mathbf{H O}_{\mathbf{a}}$ : o estado de fluxo experimentado pelos estudantes na atividade de números binários não está correlacionado com sua nota no teste de conhecimento e $\mathbf{H O}_{\mathbf{b}}$ : o estado de fluxo experimentado pelo estudante na atividade do envio de foguete a Marte não está correlacionado com sua nota no teste de conhecimento.

\subsection{Ameaças à validade}

O pequeno tamanho da amostra é considerado uma ameaça à validade externa do experimento. Novas replicações do estudo possibilitarão consolidar o conhecimento sobre o tema, em especial considerando outros perfis de estudantes, como estudantes que não sejam da área de computação e de outras faixas etárias. 
VII Congresso Brasileiro de Informática na Educação (CBIE 2018)

Anais do XXIX Simpósio Brasileiro de Informática na Educação (SBIE 2018)

Acredita-se também que a construção de um experimento com um grupo experimental e controle oferecerá um melhor indicativo sobre a eficácia (ou não) dessas duas atividades, quando comparadas à abordagens tradicionais.

\section{Resultados}

$\mathrm{Na}$ atividade de números binários, os estudantes apresentaram fluxo médio de 5,33 (em uma escala de 0 a 7), mediana de 5,44 e desvio padrão de 0,65. A média da nota na atividade foi de 8,11 , mediana 10 , e desvio padrão de 3,21 . A correlação entre o fluxo apresentado e a nota na atividade, calculada por meio do coeficiente de correlação de Pearson, não foi estatisticamente significante ( $\mathrm{p}$-value $=0,93$ ). Assim, não é possível rejeitar a hipótese $\mathbf{H O}_{\mathbf{a}}$.

$\mathrm{Na}$ atividade de envio do foguete a Marte, os estudantes apresentaram fluxo médio de 5,41, mediana de 5,55 e desvio padrão de 0,82. A média da nota na atividade foi de 8,8 , mediana 8,9 , e desvio padrão de 1,3 . A correlação entre o fluxo apresentado e a nota na atividade, calculada por meio do coeficiente de correlação de Pearson, não foi estatisticamente significante $(\mathrm{p}$-value $=0.74)$. Assim, não é possível rejeitar a hipótese $\mathbf{H} \mathbf{0}_{\mathrm{b}}$.

\section{Análise}

Os resultados do estado de fluxo indicam que os estudantes apresentaram um estado de fluxo significativo (5,33 e 5,41 em uma escala de 0 a 7) nas atividades desplugadas. $O$ pequeno desvio padrão indica que a vivência do fluxo foi relativamente uniforme entre os estudantes. Isto é bastante positivo, pois apresenta evidências estatísticas quanto à efetividade dessas atividades no engajamento dos estudantes.

Considerando a faixa etária dos estudantes de 13 a 16 anos, que possuem idade acima da recomendação das atividades oficiais, os resultados também demonstram a validade dessas atividades para essa faixa etária, ampliando suas possibilidades de uso.

Os testes de correlação não possibilitaram afirmar que o estado de fluxo possui correlação com o desempenho nas atividades de avaliação de conhecimento ( $\mathrm{p}$-value $<0.05)$. Avalia-se, no entanto, que os resultados podem ser interpretados de duas formas. A primeira interpretação é que de fato não há correlação, a segunda interpretação é que as atividades avaliativas podem ter sido simples, haja visto o alto desempenho apresentado pelos estudantes, o que pode incluir uma ameaça à validade deste resultado em específico. Sugere-se em trabalhos futuros construir e validar previamente novos instrumentos de avaliação, com o objetivo de reduzir esse possível viés.

Por fim, também foi observado que os estudantes apresentaram certa dificuldade em responder o questionário curto de fluxo, pois algumas das frases eram complexas de serem compreendidas. Este é outro fator que pode induzir um viés ao experimento e precisa ser observado com cautela por outros pesquisadores.

\section{Conclusão}

Este estudo teve por objetivo avaliar duas atividades desplugadas com estudantes do curso técnico em informática integrada ao nível médio do Instituto Federal de Pernambuco campus Garanhuns. Os resultados sugerem que as atividades apresentam potencial para 
VII Congresso Brasileiro de Informática na Educação (CBIE 2018)

Anais do XXIX Simpósio Brasileiro de Informática na Educação (SBIE 2018)

estimular o estado de fluxo nos estudantes. Por outro lado, não foi observada correlação entre o engajamento e o aprendizado, nas atividades avaliativas propostas.

Como trabalho futuro deste estudo, propõe-se replicar os experimentos realizados, com o objetivo de reduzir o viés do presente trabalho em relação ao tamanho da amostra e ao delineamento experimental. Além disto, novas atividades desplugadas serão avaliadas e espera-se assim criar um corpo de conhecimento baseado em evidências estatísticas que subsidie o trabalho docente.

\section{References}

Bell, T., Curzon, P., Cutts, Q., Dagiene, V., and Haberman, B. (2011). Overcoming obstacles to cs education by using non-programming outreach programmes. In International Conference on Informatics in Schools: Situation, Evolution, and Perspectives, pages 71-81. Springer.

Brackmann, C., Boucinha, R. M., Roman-Gonzalez, M., Barone, D. A. C., and Casali, A. (2017a). Pensamento computacional desplugado: Ensino e avaliacao na educacao primaria espanhola. In Anais dos Workshops do Congresso Brasileiro de Informatica na Educacao, volume 6, page 982.

Brackmann, C. P., Roman-Gonzalez, M., Robles, G., Moreno-Leon, J., Casali, A., and Barone, D. (2017b). Development of computational thinking skills through unplugged activities in primary school. In Proceedings of the 12th Workshop on Primary and Secondary Computing Education, pages 65-72. ACM.

Costa, T. L. S. d. (2017). O uso de computação desplugada para apoiar a aprendizagem de algoritmos de ordenação e tabela hash.

Curzon, P., McOwan, P. W., Cutts, Q. I., and Bell, T. (2009). Enthusing \& inspiring with reusable kinaesthetic activities. In ACM SIGCSE Bulletin, volume 41, pages 94-98. ACM.

Jiang, S. and Wong, G. K. (2018). Are children more motivated with plugged or unplugged approach to computational thinking? In Proceedings of the 49th ACM Technical Symposium on Computer Science Education, pages 1094-1094. ACM.

Mills, M. J. and Fullagar, C. J. (2008). Motivation and flow: Toward an understanding of the dynamics of the relation in architecture students. The Journal of psychology, 142(5):533-556.

Moura Júnior, P. J. d. et al. (2015). Se quer que seja bem feito, faça em equipe: flow e desempenho em equipes de tecnologia da informação.

Rheinberg, F., Vollmeyer, R., and Engeser, S. (2003). Die erfassung des flow-erlebens.

Rodriguez, B., Kennicutt, S., Rader, C., and Camp, T. (2017). Assessing computational thinking in cs unplugged activities. In Proceedings of the 2017 ACM SIGCSE Technical Symposium on Computer Science Education, pages 501-506. ACM. 